\title{
Analytical calculation of time of reaching specific values based on visibility loss during a fire
}

\author{
Sergei Kolodyazhniy ${ }^{1, *}$, and Vladimir Kozlov ${ }^{1}$ \\ ${ }^{1}$ Voronezh State Technical University, 20-let Oktyabrya str., 84, 394006, Voronezh, Russia
}

\begin{abstract}
Using an integral mathematical model of a fire considering the assumptions typical of a starting stage of a fire, analytical dependencies were obtained for determining the time of reaching a critical value of the density of a smoke screen in a premises with a fire epicenter and adjoining premises. By means of analytical formulas for determining critical evacuation time intervals based on visibility loss, table values for different parameters that are included in the original equations were obtained. Simple engineering analytical solutions that describe the dynamics of smoke formation in premises in case of a fire when used in a certain combination are presented. The obtained dependencies allow one to identify the critical time of evacuation with no use of special PC software as well as to obtain original data without calculating an anti-smoke ventilation system.
\end{abstract}

\section{Introduction}

In order to ensure safe evacuation from a building on fire, it is necessary to determine the time of reaching maximum acceptable temperatures, concentration of smoke, oxygen and combustion products. At the initial stage of a fire drastic changes in the average temperature, concentration of oxygen and toxic gases are uncommon. The central factor for evacuation is a time period when the density of a smoke screen is at its critical level as significant smoke formation of premises complicates navigation in space, which is detrimental for evacuation and causes a panic to unfold.

The papers [1-5] deal with mathematical models of a fire that allow for analytical solutions for determining a critical time of a fire, in [6-8] there are experimental approaches to the distribution of a smoke screen in premises with a more accurate zone model of a fire. In regards of safe evacuation in case of outbreak of a fire in one or several premises, it should be determined when a hazardous fire factor reaches its critical value both in the epicenter and adjoining premises. In this case an integral model of a fire should be employed that gives a general description of a gaseous medium as well as the most accessible one for solutions including analytical ones.

The integral model relies on a system of regular non-stationary differential equations that express the fundamental laws of a material and energy balance. This system consists of the following equations $[1,2]$ : a material balance of the gaseous medium of the premises; a material balance of the components of the gaseous medium of the premises; a material balance of the components of the gaseous medium (oxygen, carbon monoxide and dioxide,

${ }^{*}$ Corresponding authors: vgasupb@mail.ru 
chloride hydrogen and inertia gases); a material balance of smoke particles; an energy balance of the gaseous medium. There are supplementary equations that describe the mass exchange using a natural and mechanical ventilation, heat exchange between the gaseous medium and enclosing structures, combustion (gasification) of a fire load and distribution of flames along its surface. These equations are closed with the averaged equation of the current state of the medium of the premises on fire that reflects a connection of an average volumetric temperature with an average volumetric density and pressure. The initial parameters in the integral model are an average mass temperature and average volumetric pressure of the gaseous medium, average mass partial densities of oxygen, chloride hydrogen, carbon monoxide and dioxide of the gaseous medium as well as an average volumetric concentration of smoke particles.

In order to identify the integration constants during the solution process, the initial values of average volumetric parameters of the gaseous medium in the premises prior to a fire are specified. A set of the obtained regular first-order differential equations and algebraic ratios provides a mathematical description of a fire in the premises using averaged thermodynamic parameters of the state of the gaseous medium. Generally, the system of differential equations of the integral mathematical model of a fire can only be implemented by means of the numerical method and in particular, using the Runge-Kutta fourth-fifth order with a variable step. It is not possible to obtain the analytical solution of a complete non-simplified system of differential equations of a fire considering the gas exchange of the premises with the surroundings, heat exchange with the enclosing structures supplemented with the formulas of the burning rate of the combustible material and heat emission. In order to obtain the solution, some assumptions were made that are typical of the initial stage of a fire: no air coming from the surrounding medium, average pressure of the medium is constant and equals that of the outside air, smoke-forming capacity of the combustible material and coefficient of the combustion level are constant due to insignificant change in the oxygen concentration. Considering these assumptions that are proved by numerous experiments, the equation of the energy of a fire is reduced to the algebraic form and the dissolving system is reduced to four differential equations in relation to average volumetric values of the density of the gaseous medium, optical density of smoke, density of oxygen and toxic gases [1, 2]. The system of the differential equations is not coherent, the solution of each equation can be found regardless of the rest. The simplified equations of the integral mathematical model of a fire are employed to obtain the formulas for determining a critical time of a fire in the premises with the fire epicenter using the conditions of the maximum acceptable temperature, concentrations of oxygen and toxic gases as well as a critical density of smoke [1].

\section{Materials and methods}

Analytical dependencies of the integral model of a fire according to visibility loss in the premises with the fire epicenter. Mass evacuation from the premises on fire is performed at the initial stage of a fire when there is no significant change in such hazardous factors as temperature, concentrations of toxic gases and oxygen, particularly in premises adjoining those in the immediate vicinity of the fire epicenter. The central factor of a critical evacuation time is psychological impact. The time before critical amount of smoke in the premises with the fire epicenter and those adjoining it can be divided into two parts. Over the first period a critical concentration of smoke in the premises with the fire epicenter is reached and during the second one this saturated gas mass fills the adjoining premises from the ceiling down to some critical point at the floor level. Experimental studies of the distribution of smoke in different premises of buildings and structures are presented in [6-11], numerical modeling of the distribution of smoke in multi-storeyed buildings can be found in $[12,13]$. 
If we accept that the rate of sedimentation of smoke particles on the surface of the enclosing structures is a lot smaller than that of removing smoke from the premises, the differential equation for determining an average volumetric density of smoke will get dividing variables. This allows us to obtain the law for changes in time in an average volumetric density of smoke $\mu_{m}(\mathrm{~N} / \mathrm{m})$ in the analytical form [1].

$$
\mu_{c p}=\frac{D}{C}\left[1-e^{-\frac{A C}{V} \tau^{n}}\right]
$$

Here $C=\frac{\eta Q_{h}^{p}(1-\varphi)+i_{e n}}{c_{p} \rho_{0} T_{0}} ;$

$\eta$ is the coefficient of the combustion level;

$Q_{h}^{p}$ is the combustion heat, $\mathrm{J} / \mathrm{kg}$;

$\varphi$ is the heat loss coefficient;

$i_{e n}$ is enthalpy of gasification products (pyrolyze, evaporation) of the combustible material, $\mathrm{J} / \mathrm{kg}$;

$c_{p}$ is isobaric heat capacity of an ideal gas, $\mathrm{J} /(\mathrm{kg} \cdot \mathrm{K})$;

$\rho_{0}$ is the density of the medium prior to a fire, $\mathrm{kg} / \mathrm{m}^{3}$;

$T_{0}$ is the initial temperature, $\mathrm{K}$;

$D$ is smoke-forming capacity of the combustible material, $\mathrm{N} \cdot \mathrm{m}^{2} / \mathrm{kg}$;

$V$ is the volume of the premises with the fire epicenter, $\mathrm{m}^{3}$;

$A=\frac{\pi}{3} \psi_{s} v_{l}^{2}, n=3$ for circular distribution of flames;

$A=b \psi_{s} v_{l}, n=2$ for strip distribution of flames;

$\psi_{s}$ is a specific mass combustion rate, $\mathrm{kg} /\left(\mathrm{m}^{2} \cdot \mathrm{s}\right)$;

$v_{l}$ is a linear distribution rate of flames, $\mathrm{m} / \mathrm{s}$;

$b$ is the width of a strip of the distribution of flames, $m$.

This law can be employed to determine a time period when the density of smoke in the premises with the fire epicenter reaches its critical value $\mu_{c r}$. Hence for a circular distribution of flames

$$
\tau_{c r}^{s}=\sqrt[3]{\frac{V}{A C} \ln \left(\frac{D}{D-C \mu_{c r}}\right)}
$$

If the distribution of flames is along a strip with the width $b$, then

$$
\tau_{c r}^{s}=\sqrt{\frac{V}{A C} \ln \left(\frac{D}{D-C \mu_{c r}}\right)}
$$

The numerical experiment showed that the influence of the enthalpy of the gasification products of the combustible material on an average volumetric density of smoke is insignificant. Hence in the below Table 1 there are values of $\mu \mathrm{m}$ at different points in time calculated considering the value ien $=1,8 \cdot 106 \mathrm{~J} / \mathrm{kg}$ for woods and with no account of the value for other equal parameters and circular distribution of flames. The analysis of the obtained 
table data allows us to conclude that the enthalpy of the gasification products of the combustible materials (ien $=0$ ) can be neglected in further calculations.

Table 1. Calculations.

\begin{tabular}{|c|c|c|c|c|c|}
\hline$\tau, \mathrm{s}$ & 0 & 15 & 30 & 45 & 60 \\
\hline$\mu_{m}$ at $i_{e n}=1,8 \cdot 10^{6}$ & 0 & 0,0269 & 0,2127 & 0,6987 & 1,5717 \\
\hline$\mu_{m}$ at $i_{e n}=0$ & 0 & 0,0269 & 0,2132 & 0,7042 & 1,6012 \\
\hline
\end{tabular}

Fig. 1 presents a graphical dependence of an average volumetric density of smoke on time in the premises with the fire epicenter for circular distribution of flames (curved line 1) and for strip distribution (curved line 2). In the calculations it is accepted that $\eta=1, \varphi=0,5, \mathrm{cp}$ $==103 \mathrm{~J} /(\mathrm{kg} \cdot \mathrm{K}), \rho 0 \cdot \mathrm{T} 0=3 \cdot 102 \mathrm{~kg} \cdot \mathrm{K} / \mathrm{m} 3, \mathrm{~V}=60 \mathrm{~m} 3, \quad i \ni=0$; from the database [2] of a typical combustible load (furniture+household items) for a building of the I-II fire resistance level $Q_{h}^{p}=13,8 \cdot 106 \mathrm{~J} / \mathrm{kg}, \mathrm{D}=270 \mathrm{~N} \cdot \mathrm{m} 2 / \mathrm{kg}, \psi \mathrm{s}=0,0145 \mathrm{~kg} /(\mathrm{m} 2 \cdot \mathrm{s}), \mathrm{vl}=0,0108 \mathrm{~m} / \mathrm{s}$, the width of the distribution of flames $b=0,4 \mathrm{~m}$. According to the graphs, for circular distribution of flames there is a more intense growth of numerical values of an average volumetric density of smoke.

The critical distance of visibility lcr is connected with a critical value for optical density with the ratio $\mathrm{lcr}=2,38 / \mu \mathrm{cr}$.

Table 2 presents the values of time periods when in the premises on fire with the volume $\mathrm{V}=60 \mathrm{~m} 3$ for circular distribution of flames a critical distance of visibility takes the values $5,10,15$ and $20 \mathrm{~m}$ respectively. In the calculations using the formulas (2) the same values are accepted as for obtaining the graphical dependencies in Figure 1, for buildings of the I-II fire resistance level $\psi \mathrm{s}=0,0145 \mathrm{~kg} /(\mathrm{m} 2 \cdot \mathrm{s}), \mathrm{vl}=0,0108 \mathrm{~m} / \mathrm{s}$, for a building of the III-IV fire resistance level $\psi \mathrm{s}=0,0344 \mathrm{~kg} /(\mathrm{m} 2 \cdot \mathrm{s}), \mathrm{vl}=0,0465 \mathrm{~m} / \mathrm{s}, \mu \mathrm{m}, \mathrm{N} / \mathrm{m}$.

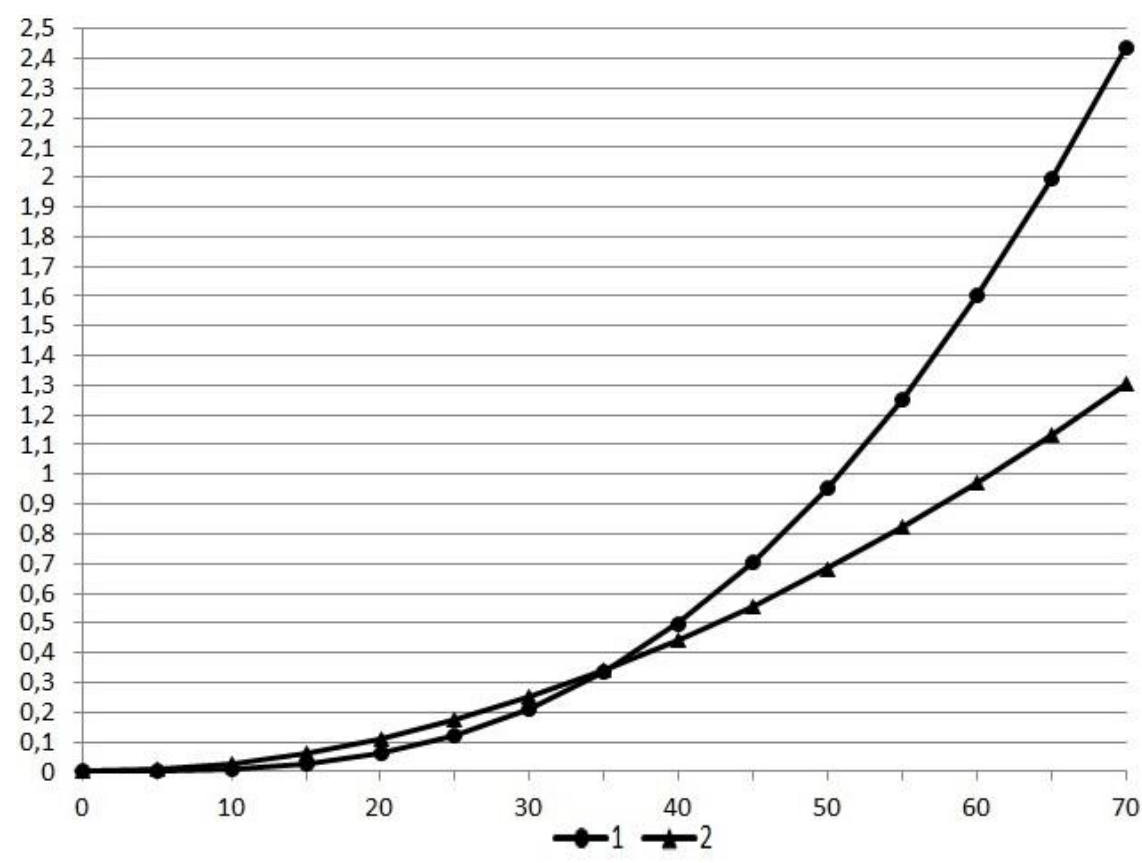

Fig. 1. $\tau$, s.

Similar data is presented in Table 3 for linear distribution of flames with a strip with the width $b=0,4 \mathrm{~m}$ for the same values that are included in the formula (3). 
Table 2. Calculations.

\begin{tabular}{|c|c|c|c|c|c|}
\hline \multicolumn{2}{|c|}{$l_{c r}$} & $5 \mathrm{~m}$ & $10 \mathrm{~m}$ & $15 \mathrm{~m}$ & $20 \mathrm{~m}$ \\
\hline \multirow{2}{*}{$\tau_{c r}^{s}$} & I-II fire resistance level & 39,4 & 31,3 & 27,2 & 24,7 \\
\cline { 2 - 6 }$(\mathrm{s})$ & III-IV fire resistance level & 11,2 & 8,8 & 7,7 & 7,0 \\
\hline
\end{tabular}

Table 3. Calculations.

\begin{tabular}{|c|c|c|c|c|c|}
\hline \multicolumn{2}{|c|}{$l_{c r}$} & $5 \mathrm{~m}$ & $10 \mathrm{~m}$ & $15 \mathrm{~m}$ & $20 \mathrm{~m}$ \\
\hline \multirow{2}{*}{$\tau_{c r}^{s}$} & I-II fire resistance level & 41,5 & 29,2 & 23,8 & 20,6 \\
\cline { 2 - 6 }$(\mathrm{s})$ & III-IV fire resistance level & 13,0 & 9,1 & 7,5 & 6,4 \\
\hline
\end{tabular}

Analytical dependencies for determining the time of visibility loss in the premises adjoining the fire epicenter. From the moment in time $\tau_{c r}^{s}$ the gases that are formed during the gas combustion will pushed the gas mass which is already on fire out of the premises with the fire epicenter through the openings into the adjoining premises (halls, adjoining rooms, etc.). As it has a higher temperature, this mix will go up to the ceiling and fill the adjoining premises while going down to the floor. The consumption of Gm coming out of the premises with the epicenter of the combustion of the gases at the moment in time in question takes up some second volume Vs $(\mathrm{m} 3 / \mathrm{s})$, with

$$
G_{m}=V_{s} \cdot \rho_{m} .
$$

In [1] considering the hypotheses for the initial stage of a fire, the equation of the energy of a fire that is identified based on the first law of thermodynamics is reduced to the algebraic form

$$
\eta Q_{h}^{p} \psi(1-\varphi)+c_{p m} T_{m} G_{m}=0 .
$$

Hence we get the expression for the second consumption of the gases that are pushed out during combustion:

$$
G_{m}=\frac{\eta Q_{h}^{p}(1-\varphi)}{c_{p m} T_{m}} \psi .
$$

In residential, household, administrative, medical, sports, cultural spaces due to openings an average pressure of the environment remains almost the same and equals that of the air outside. Then according to the algebraic equation of the averaged state of the gaseous medium in the premises

$$
\rho_{m} T_{m}=\rho_{0} T_{0}=\text { const. }
$$

Considering (7) the equality (6) can be presented as:

$$
G_{m}=\frac{\eta Q_{h}^{p}(1-\varphi)}{c_{p m} \rho_{0} T_{0}} \psi \rho_{m} .
$$

Comparing the equalities (4) and (8), we get

$$
V_{s}=\frac{\eta Q_{h}^{p}(1-\varphi)}{c_{p m} \rho_{0} T_{0}} \psi .
$$


Then over the final time period $\tau$ the mass of the pushed gases $\mathrm{M} \tau$ fills some volume $\mathrm{V} \tau$ that is given by the equality

$$
V_{\tau}=\int_{0}^{\tau} V_{s} d \tau=\frac{\eta Q_{h}^{p}(1-\varphi)}{c_{p m} \rho_{0} T_{0}} \int_{0}^{\tau} \psi d \tau
$$

The function $\psi$ in the sign of the integral has a different form depending on how a fire spreads out [2]. If a fire spreads out along the surface of solid combustible materials in a circle, then

$$
\psi=\pi \psi_{s} v_{l}^{2} \tau^{2}
$$

The mass of the combustible materials that are burnt by a moment $\tau$ is

$$
M_{\tau}=\int_{0}^{\tau} \psi d \tau=\frac{\pi}{3} \psi_{s} v_{l}^{2} \tau^{3}
$$

If flames distribute along a strip with the width $\mathrm{b} \mathrm{m}$, then

$$
\begin{gathered}
\psi=b \psi_{s} v_{l} \tau \\
M_{\tau}=\int_{0}^{\tau} \psi d \tau=\frac{b}{2} \psi_{s} v_{l} \tau^{2} .
\end{gathered}
$$

Let us present the formulas (12) and (14) with the same equality

$$
M_{\tau}=A \tau^{n}
$$

where $A=\frac{\pi}{3} \psi_{s} v_{l}^{2}, \mathrm{n}=3$ for circular distribution of flames;

$$
A=b \psi_{s} v_{l}, \mathrm{n}=2 \text { for strip distribution. }
$$

Taking into account (12), (14), (15) we get

$$
V_{\tau}=\frac{\eta Q_{h}^{p}(1-\varphi)}{c_{p m} \rho_{0} T_{0}} A \tau^{n}
$$

The equality (16) allows one to identify a time period when a gas mass with a critical density of smoke fills a critical volume Vcr of all the premises adjoining a room on fire (in a floor, in a section, etc.). This volume is calculated as the sum of the area $\mathrm{S} \Sigma$ of all the adjoining premises by the calculation height hc

$\mathrm{hc}=\mathrm{h}-\mathrm{hcr}$;

$$
V_{c r}=S_{\Sigma} \cdot h_{c}
$$

$\mathrm{h}$ is the height of the premises;

hor is a critical distance of a smoke screen to the floor level.

Inserting Vcr into the expression (16), we get a formula for determining a critical time period $\tau_{c r}^{V}$ when a dense smoke screen fills a volume Vcr

$$
\tau_{c r}^{V}=\sqrt[n]{\frac{c_{p m} \rho_{0} T_{0} S_{\Sigma} h_{c}}{A\left[\eta Q_{h}^{p}(1-\varphi)\right]}}
$$

Ultimately the critical time of evacuation takes the following form

$$
\tau_{c r}=\tau_{c r}^{s}+\tau_{c r}^{V}=\sqrt[n]{\frac{V}{A C} \ln \left(\frac{D}{D-C \mu_{c r}}\right)}+\sqrt[n]{\frac{S_{\Sigma} h_{c}}{A C}}
$$


where $C=\frac{\eta Q_{h}^{p}(1-\varphi)}{c_{p m} \rho_{0} T_{0}} ; \mu_{c r}=2,38 / l_{c r} ; \mathrm{A}$ and $\mathrm{n}$ are determined using the equality (15); in the first subradical expression of the right part $\mathrm{V}$ is a volume of the premises with an immediate fire epicenter.

The equality (18) is obtained in the assumption of filling a critical volume of all the premises adjoining the fire epicenter with a dense smoke screen from top to bottom to a critical distance to the floor level taking no account of a chaotic movement of a crowd being evacuated. It is obvious that the movement causes the upper and lower air level which is not so full of smoke to mix and thus the time $\tau_{c r}^{V}$ to increase. An analytical consideration of a chaotic movement of people that causes chaotic convective shift of the gaseous mix respectively is almost not possible. Therefore it is possible for a critical time of evacuation according to visibility loss to be determined using the above formula with a sufficient degree of accuracy.

\section{Conclusions}

Hence in this paper we presented an analytical dependence (19) for determining a critical time period when a dense smoke screen fills the entire volume of the premises adjoining those with the fire epicenter down to a critical distance to the floor level with no account of chaotic movement of a crowd being evacuated. The obtained analytical formula can be employed in engineering calculations for safe evacuation from premises in the event of a fire without using special PC software as well as for obtaining the original data for calculating an anti-smoke ventilation system.

\section{References}

1. S.A. Kolodyazhny, V.A. Kozlov, I.I. Pereslavtseva, Civil engineering and architecture 3(35), 128-138 (2014)

2. Yu. A. Koshmarov, Forecasting of dangerous factors of fire in the premises: Training manual (Moscow, 2000)

3. Yu.A. Koshmarov, V.V. Rubtsov, The process of the growth of hazards of fire in the premises and the calculation of the critical duration of the fire (Moscow, 1998)

4. I.V. Sitnikov, I.A. Shepelev, S.A. Kolodyazhny, A.A. Odnolko, Academic periodical. Engineering Systems and Structures 1, 31-35 (2012)

5. T. Daniel, T. Joseph, W. Frederick, Spill Fires, 1-36 (2012)

6. S.V. Puzach, S.A. Kolodyazhny, N.V. Kolosova, Fire and explosion safety 12(24), 33 39 (2015)

7. S.A. Kolodyazhny, N.V. Kolosova, V.A. Kozlov, K.A. Sklyarov, Journal of civil engineering and architecture 1(45), 144-156 (2017)

8. S.A. Kolodyazhnyi, S.V. Puzach, V.A. Kozlov, N.V. Kolosova, Russian Journal of Building Construction and Architecture 3(35), 89-101 (2017)

9. M. Luo, V. Beck, Fire Safety Jornal 3(26), 191-219 (1996)

10. K. Matsuyama, M. Mizuno, T. Wakamatsu, Fire Science and Technology 1(21), 43-55 (2001)

11. T. Yaman, T. Tanaka, Fire Science and Technology 1, 41-54 (1985) 
12. W.K. Chow, R. Yin, CFD simulation of air movement in a big hall induced by a bar cabin fire and studies on free boundary conditions, Journal of Fire Sciences (1998)

13. L.P. Kamenshchikov, V.I. Bykov, S.P. Amelchugov, Proc. of the 2nd Int. Seminar on Fire and Explosion Hazard of Substances and Venting Deflagrations, 650-659 (1997)

14. D. Drayzdeyl, Introduction to dynamics of the fires (Moscow, 1988)

15. H. Ruegg, T. Arvidsson, Fire safety engineering concerning evacuation from buildings 19, 45 (2009) 